\title{
Equações Diferenciais Ordinárias Fracionárias
}

\author{
A.R. Gómez Plata. \\ IMECC, UNICAMP,13083-859, Campinas, SP \\ DPTO. MATEMATICAS, UMNG, Bogotá, Colombia \\ ra142301@ime.unicamp.br, adrian.gomez@unimilitar.edu.co \\ E. Capelas de Oliveira \\ IMECC, UNICAMP \\ 13083-859, Campinas, SP \\ capelas@ime.unicamp.br.
}

\section{RESUMO}

Apresenta-se uma breve descrição das ferramentas matemáticas necessárias para entender e discutir as chamadas equações diferenciais ordinárias fracionárias, dependentes da ordem da derivada, que se constituem numa possível maneira de generalizar as equações diferenciais ordinárias. O objetivo é resolver tais equações, expressando a solução em termos das funções próprias do Cálculo Fracionário. Em particular estudamos a equação diferencial associada ao problema do chamado relaxamento (oscilações), dependendo de um parâmetro, comparando-o com a versão de ordem inteira.

Palavras-chave: Transformada de Laplace, Função gama, Equações diferenciais ordinárias de ordem não inteira, Cálculo fracionário.

\section{Introdução}

Em analogia da resolução de uma EDO, vamos utilizar neste trabalho a transformada de Laplace para resolver Equações Diferenciais Ordinárias Fracionárias (EDOF). As ferramentas matemáticas importantes para resolver uma EDOF são a função gama, funcão de Mittag-Leffler, a derivada fracionária no sentido de Caputo e Riemann-Liouville, a transformada de Laplace da derivada $\alpha$ de Caputo e RiemannLiouville, com $m-1<\alpha<m, m \in N$.

A funcão gamma é definida para $\operatorname{Re}(z)>0$,

$$
\Gamma(z)=\int_{0}^{\infty} t^{z-1} e^{-t} d t
$$

de onde, integrando por partes, temos

$$
\Gamma(z+1)=z \Gamma(z)
$$

A chamada função de Mittag-Leffler de três parâmetros, para $z \in \mathbb{C}, \beta, \rho \in \mathbb{C}$ and $R(\alpha)>0$ é

$$
E_{\alpha, \beta}^{\rho}(z):=\sum_{k=0}^{\infty} \frac{(\rho)_{k}}{\Gamma(\alpha k+\beta)} \frac{z^{k}}{k !}
$$

No caso particular, $\rho=1$, temos a função de Mittag-Leffler de dois parâmetros

$$
E_{\alpha, \beta}^{1}(z)=E_{\alpha, \beta}(z) \quad(z \in \mathbb{C})
$$


e quando $\rho=1, \beta=1$, temos a clássica função de Mittag-Leffler de um parâmetro

$$
E_{\alpha, 1}(z)=E_{\alpha}(z) \quad(z \in \mathbb{C}) .
$$

Por outro lado a integral de Riemann-Liouville de ordem $\alpha>0$ para uma função causal bem comportada $f(t)$ é dada por [1]

$$
J^{\alpha} f(t)=\frac{1}{\Gamma(\alpha)} \int_{0}^{t}(t-\tau)^{\alpha-1} f(\tau) d \tau, t>0, \alpha>0 .
$$

Vamos utilizar a derivada de Riemann-Liouville de ordem $\alpha>0$, para uma função causal bem comportada $f(t)$

$$
D^{\alpha} f(t):= \begin{cases}\frac{1}{\Gamma(m-\alpha)} \frac{d^{m}}{d t^{m}} \int_{0}^{t} \frac{f(\tau)}{(t-\tau)^{\alpha+1-m}} d \tau & m-1<\alpha<m \\ \frac{d^{m}}{d t^{m}} f(t), & \alpha=n .\end{cases}
$$

e também a chamada derivada de Caputo de ordem $\alpha>0$

$$
{ }_{c} D^{\alpha} f(t):= \begin{cases}\frac{1}{\Gamma(m-\alpha)} \int_{0}^{t} \frac{f^{m}(\tau)}{(t-\tau)^{\alpha+1-m}} d \tau & m-1<\alpha<m \\ \frac{d^{m}}{d t^{m}} f(t), & \alpha=n .\end{cases}
$$

A transformada de Laplace da derivada de Riemann-Liouville de ordem $\alpha \operatorname{com} m-1<\alpha<m$ é

$$
\begin{array}{r}
\Im\left[D^{\alpha} f(t) ; s\right]=s^{\alpha} f \overline{(s)}-\sum_{k=0}^{m-1} s^{m-1-k} g^{(k)}\left(0^{+}\right), \\
g^{(k)}\left(0^{+}\right):=\lim _{t \rightarrow 0^{+}} D^{k} g(t), g(t):=J^{(m-\alpha)},
\end{array}
$$

enquanto que a transformada de Laplace da derivada de Caputo é

$$
\begin{array}{r}
\Im\left[{ }_{c} D^{\alpha} f(t) ; s\right]=s^{\alpha} f \overline{(s)}-\sum_{k=0}^{m-1} s^{m-1-k} f^{(k)}\left(0^{+}\right), \\
f^{(k)}\left(0^{+}\right):=\lim _{t \rightarrow 0^{+}} D^{k} f(t) .
\end{array}
$$

Neste trabalho, vamos discutir a equação diferencial fracionária de ordem $\alpha>0$

$$
{ }_{c} D^{\alpha} u(t)=D^{\alpha}\left(u(t)-\sum_{k=0}^{m-1} \frac{t^{k}}{k !} u^{(k)}\left(0^{+}\right)\right)=-u(t)+q(t),
$$

com $m-1<\alpha<m$ de modo a propor exemplos, baseados nesta equação, cuja solução vem dada em termos das funções próprias do Cálculo Fracionário. Em particular estudamos a equação diferencial associada ao problema do chamado relaxamento (oscilações) fracionário, dependendo do parâmetro $\alpha$, comparando-o com a versão de ordem inteira [2].

\section{Referências}

[1] R. Gorenflo and F. Mainardi: Fractional calculus: integral and differential equations of fractional order. In: A. Carpinteri and F. Mainardi (editors): Fractals and Fractional Calculus in Continuum Mechanics. Springer Verlag, Wien and New York, 1997, pp. 223- 276.

[2] A.R. Gómez Plata: Equações Diferenciais Parciais Fracionárias. Tese de Doutorado em Mátematica Aplicada. IMECC-UNICAMP (Em andamento). 